\title{
Evaluación sobre las características del proceso de envejecimiento a través de relatos de vida*
}

\author{
Margarita Pino ${ }^{1}$ \\ Maria Carmen Ricoy ${ }^{2}$ \\ Julio Portela ${ }^{3}$
}

PINO, M.; RICOY, M.C.; PORTELA, J. Assesment of the process of ageing through life reports of elder people. Interface - Comunic., Saude, Educ., v.13, n.31, p.369-82, out./dez. 2009.

In this paper, we assess elderly people's perception of the factors that influence their health and their vital development as a starting point to improve their quality of life. We analyze 147 life stories of patients aged between 65 and 90, in an evaluative research with a biographical-narrative approach. As results and conclusions, we emphasize that the elderly reconciled their work in the industrial or services sectors with ploughing. The most common activities they perform in their free time are sewing for women and card games and watching sports on TV for men. They are satisfied with the medical assistance they receive, although they demand new specialty services and health education. Most of the patients face the deterioration of their health in a positive way.

Keywords: Self-perception of health. Quality of life. Elderly. Life stories.
En esta investigación se evalúa la percepción que presentan los mayores sobre los factores que influyen en su salud y el desarrollo vital a través del tiempo como punto de partida para mejorar su calidad de vida. Se analizan 147 relatos de vida de pacientes con edades comprendidas entre los 65 y 90 años. Se aborda una investigación evaluativa desde un enfoque biográficonarrativo. Como resultados y conclusiones hay que destacar que las personas mayores compaginaron su trabajo en el sector industrial o servicios con las labores del campo. En cuanto a las actividades que realizan en su tiempo libre destacan para las mujeres coser y para los varones los juegos de cartas y seguir los deportes por televisión. Están satisfechos con la asistencia sanitaria que reciben, aunque demandan nuevos servicios de especialidad y formación en educación para la salud. En general los pacientes se enfrentan positivamente al deterioro de la salud.

Palabras clave: Autopercepción de la salud. Calidad de vida. Personas mayores. Historias de vida.

\footnotetext{
* Este artículo se integra dentro del proyecto de investigación titulado: "Diseño de programas de intervención socioeducativa para la promoción de hábitos de salud y calidad de vida en
personas mayores" (referencia: HP20050086), subvencionado por el Ministerio de Ciencia y Tecnología español. ${ }^{1}$ Facultad de Ciencias de la Educación, Universidad de Vigo. Campus A Xunqueira, 36005 Pontevedra, España.mpino@uvigo.es ${ }^{2}$ Facultad de Ciencias de la Educación, Universidad de Vigo, España.

${ }^{3}$ Centro de Salud de Porriño, Pontevedra, España.
} 


\section{Introducción}

El envejecimiento supone un proceso dinámico que se desarrolla a lo largo de la vida. A su vez, es un proceso individual: cada persona envejece a distinta velocidad y de manera diferente. De ahí, que este proceso no se produzca por igual ni de forma uniforme para todos, ni que envejezcan en la misma persona a idéntica velocidad los distintos componentes de su organismo.

Las personas mayores pueden ser victimas de marginación social ya que pertenecen a un colectivo susceptible de exclusión (Subirats i Humet, Brugué, Gomá, 2002). Para evitar el riesgo de exclusión es necesario conocer los cambios que se producen durante este proceso vital. Los logros de la medicina, de la educación y de la ciencia en general han contribuido a que el proceso de envejecimiento tarde más en aparecer y que sea más saludable. Estos avances, que aumentan la esperanza de vida y el menor índice de natalidad en las sociedades industrializadas provocan un progresivo aumento de la población mayor de 65 años (Ribera Casado, Cruz Jentoft, 1997).

El envejecimiento es en los países industrializados el acontecimiento demográfico más importante de la época actual. Los índices de envejecimiento han crecido y continúan haciéndolo de forma vertiginosa. Estos hechos, generan una importante repercusión a nivel social y sanitario no sólo para la persona, sino también para la comunidad en la que se encuentra inmersa; lo que, lleva a pensar que este sector de la población será uno de los que demandará más servicios en los próximos años. Por eso, debemos conocer la percepción que tiene este colectivo de su propia realidad, sus carencias y necesidades con el objetivo de mejorar su calidad de vida y evitar, en lo posible, factores de riesgo (Vita, Hubert, Fries, 1998).

En el caso de España, las problemáticas referidas a la vejez son bastante recientes, ya que en 1960 sólo el 8\% de la población correspondía a personas mayores de 65 años y este porcentaje ha ido creciendo de forma ininterrumpida pasando a constituir el 15,7\% en 1997 y se prevé que ascienda hasta el $17,9 \%$ en el 2010 y al $20,3 \%$ en el 2015.

El proceso de envejecimiento varía por las distintas circunstancias que configuran la vida de cada persona: salud, ingresos económicos, familia, personalidad, tipo de trabajo, etc. La naturaleza real del proceso de envejecimiento hace difícil definir y medir cuándo una persona es anciana (Beaver, Miller, 1998). Este proceso, está influido por factores derivados de las enfermedades y padecimientos acumulados a lo largo de la vida. Todos estos elementos, así como los acontecimientos estresantes a los que ha sido sometida la persona mayor van dejando huellas a las que el organismo se tiene que adaptar (Montorio, Izal, 2000).

Si nos centramos en la perspectiva de las propias personas mayores debemos tener en cuenta que consideran que sus problemas son, por este orden: económicos, médicos, de soledad, rechazo familiar y afectivos (Vega, 1990). Su ruptura de las relaciones sociales se agrava por la pérdida de la pareja o de familiares próximos, del poder adquisitivo, la jubilación, dificultades físicas, etc. Otras carencias importantes ligadas a la vejez son la escasez de fuentes de información que manejan.

Además las personas mayores que mantienen relaciones sociales, cubren sus necesidades afectivas y emocionales, ofrecen seguridad y bienestar, acudiendo menos a la consulta médica y cuando están enfermas tienen más fuerzas y ganas de curarse (Gallo et al., 2006). En el deterioro de la calidad de vida influyen las vivencias de desarraigo y la carencia de expectativas, la soledad, el aburrimiento, el sentimiento de inutilidad y la frustración afectiva; todas estas variables pueden conducir a estados depresivos y de angustia. La soledad está relacionada con el abuso de los servicios médicos y farmacológicos, consumo de alcohol, aumento de los índices de suicidios, de la sintomatología de enfermedades físicas, del incremento de peticiones de ayuda terapéutica y social (García Pérez, Tous Ral, 1992).

Desde la preocupación social e interés profesional que produce la temática abordada y dentro de las múltiples formas de actuación existentes (sanitaria, social, educativa etc.) con la tercera edad, consideramos relevante la educación para la salud, entendida ésta como un medio para contribuir a la formación permanente de las personas mayores, abarcando los tres niveles de intervención: primaria, secundaria y terciaria.

Los programas de educación gerontológica se convierten en una herramienta básica para desarrollar experiencias de aprendizaje con las personas mayores, con la finalidad de aminorar el deterioro de su 
calidad de vida y para dar a conocer los riesgos de hábitos y conductas no saludables; así como de prácticas que mejoren o reduzcan los síntomas de determinados problemas de salud, promoviendo una implicación más activa.

La promoción de la salud empieza con las personas que están básicamente bien, ayudándoles a adoptar estilos de vida que les permitan mantener y conservar un estado aceptable de bienestar y lograr un envejecimiento lo más óptimo posible; pero también con aquellas que cuentan con factores de riesgo o que ya padecen algún tipo de enfermedad para contribuir a la mejora de su proceso terapéutico.

Desde la inquietud que nos genera la temática planteada, en este trabajo desarrollamos una investigación de carácter evaluativo para detectar las circunstancias que rodearon a un grupo de personas mayores y los efectos que originó en ellas el paso del tiempo. Analizamos la percepción que tienen los propios implicados sobre el deterioro de su salud y avance existencial, como punto de partida para el diseño de programas de educación para la salud con el objeto de contribuir a mejorar la calidad de vida del colectivo.

\section{Metodología}

Se trata de una investigación evaluativa en su modalidad descriptivo-comprensiva desde una consideración valorativa (Pérez Juste, 2006) y está desarrollada mediante el enfoque biográfico narrativo. Aunque son pocos los trabajos publicados sobre la temática abordada desde un planteamiento cualitativo (Mercado-Martínez et al., 2008) queremos sumarnos a esta iniciativa, dada la relevancia que le atribuimos a este tipo de estudios al permitirnos profundizar en peculiaridades acuciantes que rodean al colectivo objeto de análisis con el fin de describir, interpretar y comprender esta unidad contextual. Diferentes autores defienden que la presentación sistemática del enfoque biográfico narrativo, sin limitarla a la mera metodología de recogida/análisis de datos, permite construir conocimiento científico (Bolívar, 2002; Bolívar, Domingo, Fernández, 2001), visión a la que nos adherimos. Los relatos de vida que hemos recogido son narraciones autobiográficas sobre experiencias personales que poseen un interés profundo y duradero para los narradores y están contadas a través de sus propias voces (Chase, 1995); aportan evidencias fundamentalmente de carácter cualitativo sobre la percepción que tienen las personas mayores acerca de la problemática que estudiamos.

Las historias de vida propician el conocimiento sobre el contenido emocional de la experiencia humana que puede quedar ocultado por los llamados métodos objetivos. De ahí, que la investigación biográfica y narrativa se asiente, dentro del "giro hermenéutico" en una perspectiva interpretativa, en la cual las vivencias y experiencias de los participantes se convierten en el foco central de la investigación (Booth, 1996). La narrativa, por una parte, recoge la experiencia percibida y expuesta como relato por los participantes; y por otra (como enfoque de investigación), pone las pautas y formas para construir sentido a partir de acciones temporales y personales, por medio de la descripción y análisis de la información biográfica. La narración es una reconstrucción de la experiencia, por la que, mediante un proceso reflexivo, se da significado a los hechos y vivencias (Ricoeur, 1995).

Como modalidad para interpretar los materiales biográficos utilizamos los relatos producidos por un grupo de personas mayores, que como investigadores analizamos sin poner en duda su veracidad o autenticidad, con el propósito de ampliar la comprensión de la realidad examinada (Denzin, 1989). Los métodos narrativos permiten el acercamiento a las experiencias de grupos con dificultad de acceso al lograr que sus voces sean escuchadas sin subordinar la realidad de estas personas a la búsqueda de la generalización (Hornillo Araujo, Sánchez Serrano, 2003). Por tanto, entendemos que las historias de vida que hemos analizado aunque hacen referencia a una singularidad son un referente importante para este tipo de colectivos, que aunque situados en otros contextos se identifiquen de algún modo con lo sucedido.

Esta investigación narrativa en su diseño se acomete desde un estudio de caso, con el objeto de describir, interpretar y comprender la singularidad del colectivo de personas mayores analizado (Merriam, 1998). Pretendemos entender y conocer sus experiencias y vivencias desde el microcontexto estudiado, con el fin de encontrarle significado a lo largo de un recorrido vital (Rodríguez, Gil, García, 1999). 


\section{Participantes}

Los participantes objeto de estudio se concentraron en los centros de salud de las localidades de Porriño y Tomiño, situadas en el área norte de Atención Primaria de la Provincia de Pontevedra (España). En estos centros, desde el año 2002, se realiza un esfuerzo considerable por poner en marcha programas de educación para la salud destinados a personas igual o mayores de 65 años, con el fin de mejorar su estilo de vida (Pino, 2004).

En la selección se tuvieron en cuenta a las personas de 65 años y con edad superior que acudían a consulta, al menos, durante un mes y que mostraron su disponibilidad para colaborar voluntariamente en esta investigación. En total, participaron en el estudio 147 personas mayores comprendidas entre los 65 y 90 años. A todos los pacientes se les solicitó el consentimiento para recopilar y estudiar sus relatos, informándoles de la finalidad de la investigación.

Con relación a la edad, han participado en el estudio personas comprendidas entre los 65-70 años (56 mayores), de 71 a 75 años (48), entre los 76 y los 80 (31) y un pequeño grupo (12) con más de 81 años. En general (140 participantes), han vivido en algún momento de su vida en el medio rural y otros lo han hecho en núcleos urbanos en calidad de emigrante en Europa (Alemania, Suiza y Francia) o Latinoamérica (Argentina, México, Brasil y Venezuela).

En cuanto al sexo de estos participantes la distribución ha sido bastante equitativa entre las mujeres (79) y los varones (68). Por lo tanto, solamente se pensó en hacer referencia a las diferencias con relación al sexo cuando los porcentajes encontrados marquen una elevada preponderancia, en los demás casos se realizarán comentarios de modo colectivo.

Una parte importante de estas personas mayores están casadas (52 mujeres y 49 hombres) y las demás se encuentran viudas (18 mujeres y 13 hombres) o solteras ( 9 mujeres y 6 hombres).

\section{Procedimientos de análisis}

Para la recogida de información, no se ha establecido con anterioridad el número de sujetos participantes; éste se dejó abierto estimando la suficiencia de los datos a lo largo del proceso de análisis, en base a la saturación de la información obtenida. La toma de datos se simultaneó con el análisis de los mismos en un proceso interactivo y cíclico, para así determinar la conclusión de la fase de recogida de información (Marcelo, 1992).

El análisis de contenido aplicado a los relatos producidos por las personas mayores que han participado en el estudio se inicia a partir de la grabación en audio y trascripción literal de la información recogida. El análisis de esta información se desarrolló a partir del despliegue y depuración inicial, reduciendo los datos en unidades categoriales de significado para el estudio (Hernández Pina, 2001). Las categorías de análisis definidas, que se presentan en el apartado de resultados, emergen de la información recogida focalizada en el objetivo central de la investigación. El análisis de contenido de los relatos se aborda a través de una categorización general (primaria) que aglutina en su desarrollo las distintas categorías específicas fruto de la información recabada.

Las categorías definidas se determinaron a través de procedimientos de análisis cualitativos y se derivan de una concepción naturalista que aflora de los propios datos (Goetz, Lecompte, 1998). Para reforzar la validez interna del significado, en la categorización, hemos constituido los núcleos de análisis (categorías) teniendo en cuenta su consistencia a través de la configuración de elementos comunes, singulares y creíbles (Fleet, Cambourne, 1984). La fiabilidad de la codificación de las categorías fue definida por el grupo de investigadores responsables del estudio previo diálogo con los miembros del equipo de recogida de información. Éstos, habían recibido las directrices oportunas para realizar la recogida de información y guardar el anonimato de los participantes.

El recuento del porcentaje de las categorías específicas, que realizamos, no tiene como finalidad la cuantificación ni la generalización de resultados sino orientar sobre la preponderancia o singularidad de las mismas permitiendo, así mismo, profundizar en el análisis. A su vez, este recuento posibilita tener una visión general de la distribución de las categorías específicas. Además, se ofrecen al lector fragmentos ilustrativos extraídos de diferentes relatos, en el apartado de resultados, con el objeto de brindar evidencias que posibiliten ahondar en la reflexión, análisis y conclusiones. 


\section{Resultados}

En este apartado exponemos los datos obtenidos con el estudio en función de las categorías analizadas. A continuación, introducimos los respectivos subepígrafes con la categorización general (primaria) para profundizar en la interpretación de los resultados obtenidos a través de las respectivas categorías específicas.

\section{Nivel socio-económico}

En un entorno agrícola como lo es el gallego, los centros de salud se encuentran ligados geográficamente al campo, de ahí que la mayoría de las personas mayores estén vinculadas al hábitat rural y continúen desarrollando alguna tipo de labor agrícola:

"Vivíamos en el campo, trabajando mucho. Éramos seis hermanos". (Relato 45, mujer)

"Cuando era pequeña éramos cinco en casa, yo estaba con mis abuelos, vivíamos en el campo, en la aldea". (Relato 142, mujer)

Entre los participantes destaca el bajo nivel económico del que disponen durante su infancia, lo que marcó de forma determinante sus vidas ya que no pudieron estudiar y comenzaron a trabajar muy jóvenes para poder ayudar económicamente a sus familias. Por lo tanto, el nivel de estudios de estas personas mayores es precario (21,8\%), apenas saben leer y escribir; muchas de ellas $(70,1 \%)$ han realizado algún curso en los estudios primarios, aunque gran parte no ha finalizado la escolaridad obligatoria; unos pocos $(8,1 \%)$ tienen estudios secundarios y solamente uno de ellos es licenciado. Los siguientes fragmentos ilustran estos hechos:

“El nivel económico era bajo, muy bajo. Yo empecé a trabajar a los trece años en una fábrica de madera". (Relato 15, hombre)

"Un nivel económico bajo, pero bajo del todo ieh! Al no haber sueldos buenos. El campo no da para muchas cosas". (Relato 102, mujer)

Normalmente las mujeres son amas de casa (83\%) y compatibilizan este trabajo con las tareas agrícolas; mientras que los varones trabajan en el campo (45\%), en el sector servicios $(27,9 \%)$ y en el sector secundario (15\%); unos pocos $(10 \%)$ no menciona nada al respecto. En general, no consideran que sus condiciones de trabajo fuesen precarias y relatan que sus circunstancias existenciales eran aceptables.

La ayuda de los menores en las tareas del campo y del hogar convertía el absentismo escolar en una lacra sociocultural como se ilustra con el siguiente fragmento de uno de los relatos recogidos:

"No fui a la escuela, sólo unos días pero nada, tenía que ir al campo más que a otra cosa". (Relato 35)

"Fui a la escuela. Antes se iba muy tarde, a los siete años se empezaba y hasta los trece continuábamos. No iba todo el día porque a veces tenía que trabajar en el campo algo, ir a la hierba o hacer cosas de casa: lavar la ropa, ... y a lo mejor iba medio día". (Relato 32, mujer)

Como podemos observar era habitual que dejasen de ir a la escuela para ayudar a sus padres en las labores domésticas o del campo, lo que repercutió en su formación y salidas profesionales. Con una formación tan precaria, su actividad laboral estuvo centrada en tareas poco cualificadas y dispusieron de escasas posibilidades de promocionar dentro de la empresa. 
Su vida laboral activa fue intensa y dura, la mayor parte de ellos salieron de su pueblo natal e incluso emigraron a otros países para realizar distintos tipos de trabajos:

"El primer oficio fue de panadero, de aprendiz, y allí me quedé, con gran esfuerzo, hasta que hice el servicio militar. Vine y después marche a Alemania. En Alemania trabaje en una fábrica de neumáticos cerca de cuarenta años y así, ahora estoy jubilado". (Relato 87, hombre)

“Hasta los veintidós años trabajé en el campo, en casa. Después me casé y me fui para Argentina. Allí estuve de ayudante de mecánica para los barcos y luego quedamos en la calle y me fui para un taller metalúrgico. Estuve allí siete años. Tuve un negocio por mi cuenta cuando salí de navales, un mercadito. Después un amigo me dijo si quería ir con él para un bar y fui. Bien de principio, pero después mal. Vendió su parte a unos chicos. Después estuve un par de años y volví. Me dediqué a jardinero también. Después falleció la señora y fui al consulado y vi que había pasajes para el que quisiera y llevara mucho tiempo allá, se pudiera ir a España y me anote para venirme a España. Ya traía jubilación de allá". (Relato 74 , hombre)

Muchas de estas personas mayores han trabajado en diversas empresas y sectores y se jubilaron al llegar a los 65 años $(46,9 \%)$, otras $(29,3 \%)$ lo hacen de manera forzosa a causa de una enfermedad y un pequeño grupo $(12,2 \%)$ expresa que aún no se ha jubilado porque no tiene derecho a pensión contributiva. En general, los participantes $(46,3 \%)$ se alegran de jubilarse porque en este momento de sus vidas disfrutan de cosas que hasta ahora no habían podido hacer:

"Después de jubilarme bien, estoy muy contenta. Ahora debía de tener de nuevo diez años por lo menos. Muy bien porque la pensión de jubilación no es grande pero no tengo que estar trabajando y lo vamos repartiendo y vamos viviendo tranquilos". (Relato 79, mujer)

Sin embargo, un grupo (34\%) señala haber pasado momentos de tristeza al llegar la jubilación y al resto le resultó indiferente.

"Muy mal, lo lleve muy mal. Eché un año... no me pasaba el día, estaba acostumbrado a moverme y quedarme parado... era algo anormal". (Relato 56, hombre)

Otros participantes han pasado por un periodo de transición:

"Los primeros meses mal, porque estaba hecho a trabajar en el bar y todo, pero después bien. Después estuve en casa, bien, bien, bien". (Relato 38, hombre)

Hemos de señalar que las personas que hablan directamente de la jubilación son varones. Los momentos duros los relacionan fundamentalmente con la pérdida de actividad, de relaciones sociales y en otros casos con la convivencia en el hogar. Muchos emigrantes dejaban aquí a sus familias y cuando se produce el regreso se encuentran con un núcleo familiar que casi desconocen. De hecho, algunas mujeres relatan el abandono y la soledad sufrida e incluso la vida dura que han padecido al lado de su marido. Con todo, lo asumen como si se tratase de algo normal, como si fuese un simple problema familiar:

"Eran cosas que pasaban, casarte era la meta de todas, el marido podía hacer lo que quisiera, salir, entrar, emborracharse o lo que se le antojase". (Relato 50, mujer) 


\section{Actividades de la vida diaria}

Muchas de las personas mayores $(73,5 \%)$ no señalan ningún acontecimiento que haya marcado sus vidas; esto se debe a que consideran normal la pérdida de algún hijo en abortos naturales o en los primeros días de vida. La totalidad de las mujeres manifiestan que tuvieron a sus hijos en el domicilio ayudadas por la partera del lugar o sus madres.

En cuanto a las actividades que realizan en su tiempo libre, destacan para las mujeres coser (25\%) y para los hombres los juegos de cartas y ver los deportes en TV (15\%). Ambos grupos utilizan su tiempo libre, en la actualidad, para realizar algún trabajó menor en el campo y en dar paseos (19,5\%), bailar y acudir a fiestas populares $(15,7 \%)$ y, en menor medida, para hacer salidas o excursiones y cocinar, entre otras actividades.

Aquellos que no pueden desarrollar las actividades, que hemos referido anteriormente, manifiestan sentimientos de tristeza $(28,6 \%)$, resignación (13,6\%), impotencia $(6,8 \%)$, malestar $(6,8 \%)$, alegría $(29,3 \%)$ y un pequeño grupo $(15 \%)$ ni las menciona. Llama la atención, notoriamente, la respuesta de alegría que dan algunas personas al no poder realizar actividades cotidianas; esto puede deberse a que asuman con tolerancia y resignación su realidad y, por lo tanto, acepten las consecuencias que se derivan del envejecimiento; o que ésta sea una forma de evadir o esquivar el problema que representa su incapacidad.

Aquellos que no pueden trabajar en el campo señalan que les gustaría hacerlo y sienten añoranza de las labores que desarrollaron años atrás:

\footnotetext{
"Me sienta muy mal no poder ir al campo, y cuando llevo a otro (jornalero) pues es aún peor porque no lo hace como yo quería". (Relato 78, hombre)
}

Los momentos más felices que manifiestan los participantes siempre giran alrededor de sus años de juventud (o pudiera ser una revelación de la añoranza que sienten por el tiempo pasado) y en torno a acontecimientos familiares:

\footnotetext{
“Pues, los momentos más felices... mi juventud a pesar de ser así fue muy feliz porque a mi me gusta mucho la naturaleza y yo disfruto con la naturaleza, y soy muy manitas y claro gozaba. Además la franqueza que había antes, el compañerismo, antes palabra era palabra, ahora por mucho que digas... ni por escrito. Ahora todo es egoísmo, no puedes confiar en nadie". (Relato 61, hombre)

"Lo más alegre... tener los hijos y tener nietos como tengo, y alegría y tener un marido como tengo que es un sol". (Relato 41, mujer)
}

Una información que llama la atención, es que nuestros mayores pocas veces nombran como personas allegadas a ellos aquellas que no pertenecen a su núcleo familiar; pero, sin embargo, al comentar con quien realizan las actividades de ocio observamos que normalmente lo hacen con personas con las que no les une ningún parentesco, fundamentalmente con vecinos y amigos.

\section{Cuidados y atención sanitaria}

En la actualidad, muchos de los mayores que participan en el estudio (46,9\%) manifiestan que se enfrentan positivamente al deterioro de la salud; otros $(36,7 \%)$ indican que lo afrontaron negativamente, con paciencia (15\%) o con nerviosismo y ansiedad (1,4\%). En general, las enfermedades que padecen están relacionadas con una degeneración ósea, hipertensión, hiperlipemia, diabetes o tabaquismo en los varones.

Gran parte de los mayores echan de menos el apoyo familiar sobre todo cuando sufren alguna enfermedad: 
“Tengo a mis hijos trabajando todos, y aunque no viven lejos no vienen por aquí a no ser que uno esté muy mal". (Relato 122, mujer)

Otros participantes están unidos a sus vecinos para evitar la soledad:

“Los vecinos están para todo, para llevarte al médico, para echar una parrafada, para lo que sea, Yo le tengo muy buenos vecinos la verdad". (Relato 34, mujer)

En otros casos, acuden directamente al centro de salud:

“Desde que tenemos el centro de salud, todo está mucho mejor. Puedes acudir allí si tienes algún problema. Son muy amables y ayudan en todo lo que pueden". (Relato 145, hombre)

Encontramos que los momentos más tristes para las personas mayores son consecuencia de diferentes episodios ligados a las desgracias personales o familiares, enfermedades que padecen, pérdidas familiares etc.:

"Triste es que mi mujer está bastante mal, en fin, bebe algo". (Relato 55, hombre)

"Cuando mi mujer murió, me quede sólo, mis hijos fuera. Yo no sabía hacer nada sin ella, ni ir al banco". (Relato 126, hombre)

"Los momentos más tristes son cuando me pongo enfermo, nunca me encuentro como debo de encontrarme". (Relato 98, hombre)

En general (80\%), nuestros mayores están muy satisfechos con el sistema sanitario del que disponen y con los servicios que le ofrece actualmente; y un pequeño grupo manifiesta una menor complacencia. Algunos mayores reivindican nuevos servicios para sus centros de salud, sobre todo de especialidad médica:

"Que hubiera un médico que mirara todo. Un especialista que fuera bueno". (Relato 56, mujer)

"Me gustaría que hubiese radiología, por ejemplo, y la rehabilitación también porque no creo que sea tan difícil ni tan costoso". (Relato 46, mujer)

"Tenía que haber todos los servicios para no tener que trasladarse. También que hubiera charlas, cosas relacionadas con la salud para nosotras... me gustaría mucho. Yo participaría". (Relato 58, mujer)

Las personas mayores $(95,2 \%)$, están contentas con la función que desarrolla el personal sanitario y el trato que se le da en los centros de salud públicos. En sus relatos, las personas mayores también manifiestan que les gustaría implicarse en actividades promovidas por los centros de salud para mejorar su calidad de vida (83\%).

A pesar de que la utilización que realizan los participantes de los servicios sanitarios es excesiva, hemos de precisar que la mayoría de las visitas no se deben a patológicas, sino para resolver problemas burocráticos (recetas en el caso de enfermos crónicos, documentos para tramitar la pensión, anotarse a las excursiones organizadas por el Instituto de Servicios Sociales y para acudir a los balnearios subvencionados), problemas sociales (soledad, abandono y pobreza), problemas psicológicos (angustia y depresión) o problemas educativos (por no saber utilizar correctamente su medicación, no seguir las pautas de alimentación de acuerdo con su enfermedad o desconocer que actividades físicas pueden practicar). 


\section{Autopercepción de la salud}

Un grupo de personas mayores (49\%) considera que su salud es regular; mientras otro la perciben como buena (32,4 \%); y una minoría como muy buena (5,9\%), muy mala (2\%) o mala (10, $8 \%$ ). Algunos mayores manifiestan que sienten dolor casi todos los días $(34,4 \%)$, mientras otros indican que no sufren dolor (37,3 \%) y unos pocos expresan que lo padecen algún día por semana. En general, en los relatos lo más revelador, sí no existen enfermedades muy graves, es que la etapa de la jubilación es la más apacible de su vida.

“Esta es la mejor época de nuestra vida. Podemos hacer lo que queramos y tenemos muchas oportunidades: bailes, excursiones, los amigos. Además tenemos todo el tiempo. A mejorado mucho la cosa, nuestros padres no pudieron disfrutar nada de esto". (Relato 59, mujer)

Esta percepción positiva sobre la salud tiene relación con el nivel de independencia física, y también económica con el que cuentan, gracias a las pensiones, a la ausencia de enfermedades invalidantes y al apoyo social, sobre todo de la familia. De su vejez valoran fundamentalmente la libertad de la que disponen para realizar muchas cosas para las que antes no tenían tiempo, aunque sus años más felices los relacionan con la juventud.

"Ahora las cosas van mucho mejor, si estamos bien podemos salir y hacer muchas actividades en el ayuntamiento, en el centro de salud, y también en el centro social. La cuestión es no perder la cabeza y poder andar bien". (Relato 76, hombre)

De los relatos recogidos, se deduce que los participantes consideran su bienestar, más como una postura ante la vida que la consecuencia de su estado físico. Se aprecia una percepción positiva ante la vida cuando mantienen sus relaciones sociales, sienten el apoyo familiar y también de los amigos, y poseen una independencia económica que les facilita tomar decisiones libremente sobre su ocio.

En algunos casos (13\%), denuncian su malestar por la imagen negativa que tiene de ellos la sociedad:

"Piensan que no servimos para nada. Mis nietos si digo algo ni me oyen, les parece que ya no sirve y lo que digo son tonterías". (Relato 53, hombre)

Una valoración negativa ante la vida, como es natural, es más acuciante en los participantes cuyas enfermedades les producen un dolor pronunciado o en los que han perdido a su conyugue después de muchos años de convivencia.

Las personas mayores a pesar de considerar que poseen buena salud para la edad que tienen, en general, utilizan los servicios sanitarios como mínimo dos veces al mes. Este hecho, lo justifican señalando que deben de ir a recoger las recetas de medicamentos debido a enfermedades crónicas que padecen, por vacunaciones; curiosamente en algunos casos señalan el centro de salud como lugar de encuentro y conversación.

"Ya quedamos los martes para ir al centro de salud y así nos acercamos al mercado y ya volvemos juntos en el taxi. Además si llueve o hace frío allí hay calefacción y se está bien". (Relato 137, mujer)

Descubrimos con las manifestaciones de los participantes, a través de los relatos, que aquellas personas que tienen menos relaciones sociales visitan más al médico que las que desarrollan diferentes actividades y, por lo tanto, su red social suele ser más amplia y mantienen el tiempo ocupado. En concreto, visitan más al médico los que no realizan actividades de ocio, no viven con familiares directos y en algunos casos hasta se llevan mal con los vecinos (4 casos). 
Los participantes, en muchos casos, no acuden al médico para buscar un diagnóstico, sino más bien para pedir consejo:

"A veces voy porque estoy que no respiro, que me falta el aire, y no sé que hacer. Voy allí y el doctor me dice lo que tengo que hacer con mi hija y mis nietos que solo me dan disgustos. Si no le hiciera caso. Cada uno es como es". (Relato 82, mujer)

Algunas personas mayores tienen la falsa creencia de que los avances científicos actuales pueden dar solución a cualquier problema de salud:

"Ahora con los avances nuevos que hay se puede vivir mucho mejor. Si en el centro de salud no hay técnicas nos mandan al hospital que hay de todo y te arreglan lo que haga falta. Y si tienes que morir te mueres. Si te viene un cáncer no hay nada que hacer". (Relato 112, hombre)

\section{Conclusiones y discusión}

Las personas mayores consideran aceptables las condiciones duras de vida, que han padecido, desde su juventud a pesar de las penurias sufridas porque en el entorno en que vivían estaban normalizadas, ya que todos soportaban calamidades. Algunos estudios ponen de manifiesto que las memorias acumulativas de un lugar permiten a las personas mantener una autoimagen favorable a pesar de las contingencias de su vida posterior (Taylor, 2001). De hecho, se ha detectado en otro estudio una tendencia del anciano por sentirse satisfecho con la propia historia de vida (Molina Sena, Meléndez Moral, 2007), aunque ésta también puede ser una forma engañosa de autoconsuelo o desahogo.

El trabajo en el campo continúa formando parte de la vida de nuestros mayores, incluso como ocio, y lo compatibilizan con otras actividades como excursiones, juegos, bailes, etc. Normalmente estas actividades las realizan con amigos, familiares o vecinos. Sin embargo, se constata que la mayoría de las relaciones sociales se producen con los vecinos. En otra investigación encuentran que los mayores sienten la obligación de incluir a familiares en su red social (Piñón Paya, 1999); de hecho integran parientes con los que apenas mantuvieron contacto y presentan reticencia a incorporar personas con las que frecuentemente pasaban muchas horas.

Las personas mayores consideran que tienen buena salud y sólo les angustia la falta de autonomía y el deterioro de la capacidad cognitiva. De hecho, la percepción positiva de la salud parece estar más relacionada con el mantenimiento de una red de apoyo social o una independencia económica que con el estado físico. Resulta evidente en el estudio la importancia de mantener las relaciones sociales basadas fundamentalmente en sus familiares directos, pero también en sus vecinos y amigos. En algunas investigaciones se observó una correlación inversa entre apoyo familiar y número de enfermedades (Salgado de Zinder, 2005).

La percepción negativa que tienen sobre su salud algunas personas mayores parece estar asociada al padecimiento de enfermedades que producen un dolor importante o a la pérdida de las relaciones sociales. De hecho, algunos estudios señalan que la calidad de vida en la vejez se supedita a disponer de salud porque supone independencia, funcionalidad y además redes de apoyo familiar y social (Ors, Laguna, 1997).

A través de otro estudio cualitativo se ha llegado a la conclusión de que entre las variables generadoras de bienestar destacan los elementos familiares y sociales como las categorías más satisfactorias, frente a los elementos de seguridad como los más insatisfactorios (Molina Sena, Meléndez Moral, 2007). Además, es relevante indicar que la soledad tiene una posición prevalente en las mujeres frente a los varones. Tradicionalmente, era en el ámbito familiar y privado donde la persona mayor encontraba cobijo y amparo gracias a la labor en el hogar de la mujer, actualmente con su incorporación al mercado laboral disminuye la dedicación (Pascual Monzó, 2001). Otro aspecto a tener en cuenta con 
personas mayores de 65 años es la visión que tienen sobre su salud, dado que el riesgo de mortalidad se reduce en las optimistas (Van Doorn, 1999).

\section{Algunas sugerencias}

Con el apoyo pertinente de los organismos públicos y/o privados adelantamos como ejes pedagógicos asociados a la promoción de la calidad de vida con personas mayores algunas líneas de actuación:

- Mantener, ampliar y dinamizar la red de apoyo social con el fin de que se sientan útiles en su comunidad y mantengan los vínculos de unión que le permitan considerarse miembro de la misma, para evitar el desarraigo y la soledad. Para ello, es importante identificar, mediante un protocolo de actuación los problemas más característicos sobre la salud de la población mayor.

- Crear cauces de participación comunitaria, para las personas mayores, que tengan como meta organizar y movilizar a este colectivo, desde el propio coprotagonismo de los mayores: adquiriendo sentido de responsabilidad sobre su propio bienestar y el del colectivo; tomando parte en las deliberaciones y decisiones de problemáticas que afecten a la comunidad; participando en iniciativas sobre planes y adopción de medidas para atender a sus necesidades y expectativas, así como a la evaluación sobre el grado de satisfacción y resultados.

- Favorecer la conservación de la capacidad funcional, ya que incide de forma determinante en su autonomía e independencia, así como la integridad psicológica con programas de estimulación cognitiva; y actividades de mantenimiento físico y motriz aprovechando las rutinas cotidianas de las personas mayores. Por ejemplo, memorizar la lista de la compra, realizar otras tareas sencillas en el hogar, retener o aprender la letra de sus canciones favoritas o pasear a diario en grupo.

- Proporcionar nuevas ofertas de actividades para el tiempo libre. Es un hecho la repercusión negativa que sobre la salud y el bienestar de las personas mayores tiene el disponer de mucho tiempo de ocio sin utilidad alguna (Auer, 1997); señalándose que éste puede ser consecuencia de la falta de estímulos adecuados (Laforest, 1991). En este punto, consideramos que los programas de tiempo libre deben partir de las necesidades contextuales en las que se encuentran inmersos los mayores, sus intereses y posibilidades, integrando armónicamente sus dificultades con las potencialidades que presentan (Ricoy, 2004).

- Diseñar planes para la intervención preventiva primaria mediante estrategias educativas que favorezcan la adopción de hábitos saludables. Estos programas pueden llevarse a cabo en los centros educativos o de salud, en las asociaciones culturales u otros organismos mediante diferentes técnicas participativas: encuentros, intercambios, charlas, cursos, talleres etc.

\section{Colaboradores}

Margarita Pino y Maria Carmen Ricoy, han sido responsables y colaboraron conjuntamente en el desarrollo de la totalidad del artículo elaborado. Julio Portela, colaboró en el asesoramiento médico. 


\section{Referencias}

AUER, A. Envejecer bien: un estímulo ético-teológico. Barcelona: Herder, 1997.

BEAVER, M.L.; MILLER, D.A. Práctica clínica del trabajo social con personas mayores. Buenos Aires: Paidós, 1998.

BOLÍVAR, A. ¿De nobis ipsis silemus?: epistemología de la investigación biográficonarrativa en educación. Redie, v.4, n.1, 2002. Disponível em: <http://redie.uabc.mx/ vol4no1/contenido-bolivar.html>. Acceso en: 23 jul. 2008.

BOLÍVAR, A.; DOMINGO, J.; FERNÁNDEZ, M. La investigación biográfico-narrativa en educación: enfoque y metodología. Madrid: La Muralla, 2001.

BOOTH, T. Sounds of still voices: issues in the use of narrative methods with people who have learning dificulties. In: BARTON, L. (Ed.) Disability and society: emerging issues and insights. New York: Logman Publishing, 1996. p.237-55.

CHASE, S.E. Taking narrative seriously: consequences for method and theory in interview studies. Narrat. Stud. Lives, v.3, n.1, p.1-26, 1995.

DENZIN, N.K. Interpretative biography. Newbury: Sage, 1989.

FLEET, A.; CAMBOURNE, B. The coding of naturalistic data. Res. Educ., n.41, p.1-15, 1984.

GALLO, J.J.; BOGNER, H.R.; FULMER, T.; PAVEZA, G.J. Handbook of geriatric assessment. Maryland: Aspen Publication, 2006.

GARCÍA PÉREZ, M.C.; TOUS RAL, J.M. Motivación y vejez. Barcelona: Fundación “La Caixa", 1992.

GOETZ, J.P.; LECOMPTE, M.D. Etnografía y diseño cualitativo en investigación educativa. Madrid: Morata, 1998.

HERNÁNDEZ PINA, F. Bases metodológicas de la investigación educativa. Murcia: Diego Marín, 2001.

HORNILLO ARAUJO, E.; SÁNCHEZ SERRANO, J.L. El interés emergente por la narrativa como método en el ámbito socio-educativo: el caso de las historias de vida. Portularia, n.3, p.373-82, 2003.

LAFOREST, J. Introducción a la gerontología. Barcelona: Herder, 1991.

MARCELO, C. Dar sentido a los datos: la combinación de perspectivas cualitativas y cuantitativas en el análisis de entrevistas. In: MARCELO, C. (Coord.). La investigación sobre la formación del profesorado: métodos de investigación y análisis de datos. Argentina: Cincel, 1992. p.13-48.

MERCADO-MARTÍNEZ, F.; TEJADA-TAYABAS, L.M.; ALCANTARA-HERNANDEZ, E.; MERCADO-MARTINEZ, A.; FUENTES-URIBE, I.X.; TRIGUEROS-BECERDA, B. Enseñando investigación cualitativa en salud: evaluación de un curso de formación en la perspectiva de los alumnos. Interface - Comunic., Saude, Educ., v.12, n.26, p.515-26, 2008.

MERRIAM, S.B. Qualitative research and case de study applications in education. San Francisco: Jossey-Bass Publishers, 1998.

MOLINA SENA, C.J.; MELÉNDEZ MORAL, J.C. Análisis cualitativo del bienestar en la vejez. Rev. Esp. Geriatr. Gerontol., v.42, n.5, p.276-84, 2007.

MONTORIO, I.; IZAL, M. Intervención psicológica en la vejez. Madrid: Síntesis, 2000.

ORS, A.; LAGUNA, A. Reflexiones sobre el envejecimiento y la calidad de vida. Cult. Cuid., v.1, n.2, p.60-3, 1997. 
PASCUAL MONZÓ, R.M. Los mayores en Galicia: reflexiones a propósito de una investigación acerca de sus necesidades. In: FUNDACIÓN CAIXA GALICIA. Familia, juventud y nuestros mayores: la actitud preactiva. A Coruña: Fundación CaixaGalicia, 2001. p.402-15.

PÉREZ JUSTE, R. Evaluación de programas educativos. Madrid: La Muralla, 2006.

PINO, M.R. El deterioro de la salud como factor de riesgo de desadaptación social. In: ALBERTE CASTIÑEIRAS, J.R. (Coord.). Atención educativa à diversidade e políticas de integración. Santiago de Compostela: Universidade de Santiago de Compostela-ICE, 2004. p.105-28.

PIÑÓN PAYÁ, M. Los cambios en la red social y en la percepción de la salud durante la jubilación. 1999. Tesis (Doctoral) - Departamento de Antropología Social y Filosofía, Universidad Rovira y Virgili, España. 1999.

RIBERA CASADO, J.M.; CRUZ JENTOFT, A.J. Geriatría en atención primaria. Barcelona: Uriach, 1997.

RICOEUR, P. Tiempo y narración I: configuración del tiempo en el relato histórico. Madrid: Siglo XXI, 1995.

RICOY, M.C. Los modelos de formación como medios para contribuir al desarrollo de las múltiples dimensiones de las personas adultas. In: CARDONA, J. (Coord.). La calidad territorial como estrategia: la formación, clave en el desarrollo. Talavera de la Reina: UNED, 2004. p.127-43.

RODRÍGUEZ, G.; GIL, J.; GARCÍA, E. Metodología de la investigación cualitativa. Málaga: Aljibe, 1999.

SALGADO DE ZINDER, V.N.; GONZÁLEZ-VAZQUEZ, T.T.; JÁUREGUI-ORTIZ, B.; BONILLA-FERNÁNDEZ, P. No hacen viejos los años, sino los daños: envejecimiento y salud en varones rurales. Salud Publica Mex., v.4, n.47, p.294-302, 2005.

SUBIRATS I HUMET, J.; BRUGUÉ, Q.; GOMÁ, R. De la pobreza a la exclusión social: nuevos retos para las políticas públicas. Rev. Int. Sociol., n.33, p.7-45, 2002.

TAYLOR, S.A. Place identification and positive realities of aging. J. Cross Cult. Gerontol., v.16, n.1, p.5-20, 2001.

VAN DOORN, C. Qualitative approach studying health optimism, realism and pessimism. Res. Aging, v.3, n.21, p.440-57, 1999.

VEGA, J.L. Psicología de la vejez. Salamanca: Cervantes, 1990.

VITA, A.J.; TERRY, R.B.; HUBERT, H.B.; FRIES, J.F. Aging, health risks, and cummulative disability. N. Engl. J. Med., n.338, p.1035-41, 1998. 
PINO, M.; RICOY, M.C.; PORTELA, J. Avaliação sobre as características do processo de envelhecimento por meio de histórias de vida. Interface - Comunic., Saude, Educ., v.13, n.31, p.369-82, out./dez. 2009.

Avalia-se a percepção que apresentam as pessoas idosas sobre os fatores que influenciam sua saúde e seu desenvolvimento vital como ponto de partida para melhorar sua qualidade de vida. São analisadas 147 histórias de vida dos pacientes com idades entre 65 e noventa anos, em uma investigação avaliativa com abordagem biográficonarrativa. Como resultados e conclusões, ressalta-se que as pessoas idosas conciliaram seu trabalho no setor industrial, ou de serviços, com a lavoura no campo. Quanto às atividades que realizam no seu tempo livre, destacam-se a costura para as mulheres e os jogos de cartas e a programação desportiva da televisão para os homens. Estão satisfeitos com a assistência que recebem na saúde, embora demandem novos serviços de especialidade e formação na educação para a saúde. De um modo geral, os pacientes defrontam-se com a deterioração da saúde positivamente.

Palavras-chave: Autopercepção da saúde. Qualidade de vida. Pessoas idosas. Histórias de vida.

Recebido em 23/10/08. Aprovado em 19/07/09. 